\title{
Silicone Rubber Fabry-Perot Pressure Sensor Based on a Spherical Optical Fiber End Face
}

\author{
Changxing Jiang, Xiaohua Lei *, Yuru Chen, Shaojie Lv, Xianming Liu and Peng Zhang
}

Citation: Jiang, C.; Lei, X.; Chen, Y.; Lv, S.; Liu, X.; Zhang, P. Silicone Rubber Fabry-Perot Pressure Sensor Based on a Spherical Optical Fiber End Face. Sensors 2022, 22, 1862. https://doi.org/10.3390/s22051862 Academic Editors: Sylvain Girard, Jeroen Missinne, Geert Van Steenberge and Thomas Geernaert

Received: 21 January 2022

Accepted: 24 February 2022

Published: 26 February 2022

Publisher's Note: MDPI stays neutral with regard to jurisdictional claims in published maps and institutional affiliations.

Copyright: (C) 2022 by the authors. Licensee MDPI, Basel, Switzerland. This article is an open access article distributed under the terms and conditions of the Creative Commons Attribution (CC BY) license (https:// creativecommons.org/licenses/by/ $4.0 /)$.
Key Laboratory of Optoelectronic Technology \& Systems, Education Ministry of China, Chongqing University, Chongqing 400044, China; 201908021007@cqu.edu.cn (C.J.); chenyuru@cqu.edu.cn (Y.C.); 20172469@cqu.edu.cn (S.L.); xmliu@cqu.edu.cn (X.L.); zhangpeng@cqu.edu.cn (P.Z.)

* Correspondence: xhlei@cqu.edu.cn

\begin{abstract}
To improve the fringe contrast and the sensitivity of Fabry-Perot (FP) pressure sensors, a silicone rubber FP pressure sensor based on a spherical optical fiber end face is proposed. The ratio of silicone rubber ingredients and the diameter and thickness of silicone rubber diaphragm were optimized by a simulation based on experimental tests that analyzed elastic parameters, and the influence of the radius of a spherical optical fiber and the initial cavity length of the sensor on the fringe contrast was investigated and optimized. Pressure sensor samples were fabricated for pressure test and temperature cross-influence test. Gas pressure experimental results within a pressure range of $0 \sim 40 \mathrm{kPa}$ show the average sensitivity of the sensor is $-154.56 \mathrm{~nm} / \mathrm{kPa}$ and repeatability error is less than $0.71 \%$. Long-term pressure experimental results show it has good repeatability and stability. Temperature experimental results show its temperature cross-sensitivity is $0.143 \mathrm{kPa} /{ }^{\circ} \mathrm{C}$. The good performance of the proposed FP pressure sensor will expand its applications in biochemical applications, especially in human body pressure monitoring.
\end{abstract}

Keywords: pressure; Fabry-Perot sensor; silicone rubber; spherical optical fiber

\section{Introduction}

The optical fiber FP pressure sensor has many advantages, such as anti-electromagnetic interference, small size, etc. It has been gradually used in aerospace, bridge detection, large instrument, equipment monitoring, etc. [1-4]. Recent studies show it has also potential biochemical applications, especially in human body pressure monitoring [5-9]. For example, Shen Liu et al. proposed an arc discharge technology to produce an ultrathin silica diaphragm with a thickness of only $173 \mathrm{~nm}$, and its pressure sensitivity is $12.22 \mathrm{~nm} / \mathrm{kPa}$ [10] $\mathrm{Xu}$ Guo et al. proposed a thermal bonding technology, in which only a $1.2 \mu \mathrm{m}$ thickness silicon dioxide diaphragm bonded to the end face of single-mode fiber, where there is an etched microcavity. Additionally, its static pressure sensitivity is $12.4 \mathrm{~nm} / \mathrm{kPa}$ [11]. In addition, a few layers of graphene material has also been used to improve the pressure sensitivity to $39.4 \mathrm{~nm} / \mathrm{kPa}$ [12]. It can be seen that the effective way to achieve high-pressure sensitivity is to reduce the thickness of the diaphragm. However, the excessively thin diaphragm is too fragile, which makes the manufacturing process difficult, significantly increases manufacturing costs, and reduces its reliability [13-17].

To solve this problem, some FP sensors with diaphragms based on new elastic materials have been reported. To date, Jushuai $\mathrm{Wu}$ et al. has reported the smallest FP pressure sensor, with a SU-8 photoresist directly coated on the end face of a single-mode optical fiber. It has a support structure diameter of $100 \mu \mathrm{m}$ and a diaphragm thickness of $11 \mu \mathrm{m}$ and shows a sensitivity of $2.93 \mathrm{~nm} / \mathrm{MPa}$ in the range of $0 \sim 700 \mathrm{kPa}$ [18]. Cheng Luo et al. used a PDMS polymer as the pressure sensing diaphragm; its thickness is $18.4 \mu \mathrm{m}$ and pressure sensitivity is $100 \mathrm{pm} / \mathrm{kPa}$ [19]. Xin Cheng et al. investigated an FP pressure sensor based on a polymer of the silicone rubber diaphragm, with a thickness of $257 \mu \mathrm{m}$, and its pressure sensitivity is $0.69 \mathrm{~nm} / \mathrm{kPa}$ [20]. From these studies, we can infer that the thickness of the 
polymer diaphragm can be 1-2 orders higher than that of the traditional diaphragm under the same sensitivity. Obviously, a thicker polymer diaphragm makes the manufacturing process easier. However, the FP sensors with polymer diaphragms also have low sensitivity and a low precision caused by the poor fringe contrast [21-23].

In this paper, we proposed a silicon rubber fiber FP pressure sensor based on a spherical end fiber. An FP cavity was formed by a spherical optical fiber end face and the silicone rubber surface. A convergence effect of the spherical surface increased the intensity of the reflected light from the rubber surface returning to the optical fiber, so the fringe contrast of the sensor greatly improved. Additionally, the sensitivity of the sensor also improved by optimizing the ratio of silicone rubber ingredients.

\section{Fringe Contrast Analysis of FP Pressure Sensors}

For a typical optical fiber FP pressure sensor, its optical path is shown in Figure 1. The incident light is $\vec{E}_{0}(\lambda)$; a part of the light reflected by the first surface $A$ is recorded as $\vec{E}_{1}(\lambda)$, and the transmitted light reflected by the second surface $B$ and then transmitted through the first surface is $\vec{E}_{2}(\lambda)$. The light reflected by surface $B$ (pressure sensing diaphragm) cannot be completely coupled back to the optical fiber core because of the limited numerical aperture of optical fiber. As the cavity length $L$ increases, the light that can be coupled back to the core decreases. When the diameter of the diaphragm is equivalent to the diameter of the optical fiber, the pressure applied on the diaphragm will increase its curvature, which will increase the loss of light coupled back into the fiber. This increases the energy difference between the $\vec{E}_{2}(\lambda)$ and the $\vec{E}_{1}(\lambda)$, reducing the fringe contrast of interference patterns and decreasing demodulation accuracy $[24,25]$.

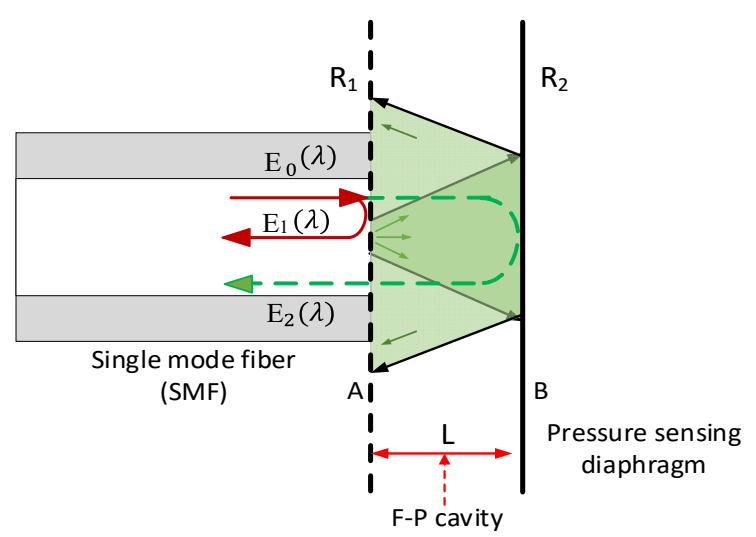

Figure 1. The optical path diagram of the optical fiber FP pressure sensor.

Suppose the transmission coefficient is $\eta$, which describes the total reflection loss from surface $B$ to the optical fiber core. Effective reflectivity is defined as $\eta R_{2}=\eta r_{2}^{2}$, and expressions of $\vec{E}_{1}(\lambda)$ and $\vec{E}_{2}(\lambda)$ can then be written as Equations (1) and (2).

$$
\begin{gathered}
\vec{E}_{1}(\lambda)=\sqrt{R_{1}} \vec{E}_{0}(\lambda) \\
\vec{E}_{2}(\lambda)=\left(1-\sqrt{R_{1}}\right)^{2} \eta \sqrt{R_{2}} \vec{E}_{0}(\lambda) \cdot \exp \left(j \frac{4 \pi L}{\lambda}\right)
\end{gathered}
$$

The total electric field $\vec{E}_{R}(\lambda)$ can be expressed as

$$
\vec{E}_{R}(\lambda)=\vec{E}_{1}(\lambda)+\vec{E}_{2}(\lambda)=\left[\sqrt{R_{1}}+\left(1-\sqrt{R_{1}}\right)^{2} \eta \sqrt{R_{2}} \cdot \exp \left(j \frac{4 \pi L}{\lambda}\right)\right] \cdot \vec{E}_{0}(\lambda)
$$


Then, the intensity $I_{R}(\lambda)$ can be written as

$$
I_{R}(\lambda)=\vec{E}_{R}(\lambda) \cdot \vec{E}_{R}^{*}(\lambda)=\left[\frac{R_{1}+\eta R_{2}+2 \eta \sqrt{R_{1} R_{2}} \cos \frac{4 \pi L}{\lambda}}{1+\eta R_{1} R_{2}+2 \eta \sqrt{R_{1} R_{2}} \cos \frac{4 \pi L}{\lambda}}\right] \cdot I_{0}(\lambda)
$$

Let the fringe contrast of interference pattern be

$$
K=\frac{I_{R_{\max }}-I_{R_{\min }}}{I_{R_{\max }}+I_{R_{\min }}}
$$

To further understand the relationship between the transmission coefficient and the fringe contrast, a simulation was carried out based on Equations (4) and (5), which is shown in Figure 2. The fringe contrast decreases with the decrease in transmission coefficient because of the high reflection loss caused by the change in the cavity length $L$ or the diaphragm curvature. The reduction in fringe contrast leads to the deterioration of the signal-to-noise ratio and low demodulation accuracy. Therefore, it is necessary to investigate a new sensor structure to increase the intensity of $\vec{E}_{2}(\lambda)$ so that the fringe contrast can be improved.

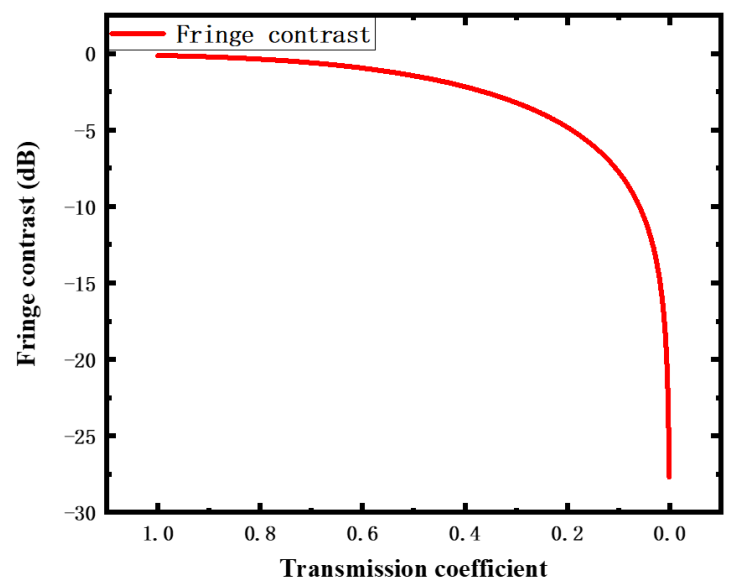

Figure 2. Relationship between transmission coefficient and fringe contrast of interference pattern.

\section{Sensor Design Based on a Spherical End Face}

\subsection{Sensor Structure}

In order to improve the fringe contrast, a silicone rubber FP pressure sensor structure based on a spherical end face was proposed, and the sensor structure is shown in Figure 3.

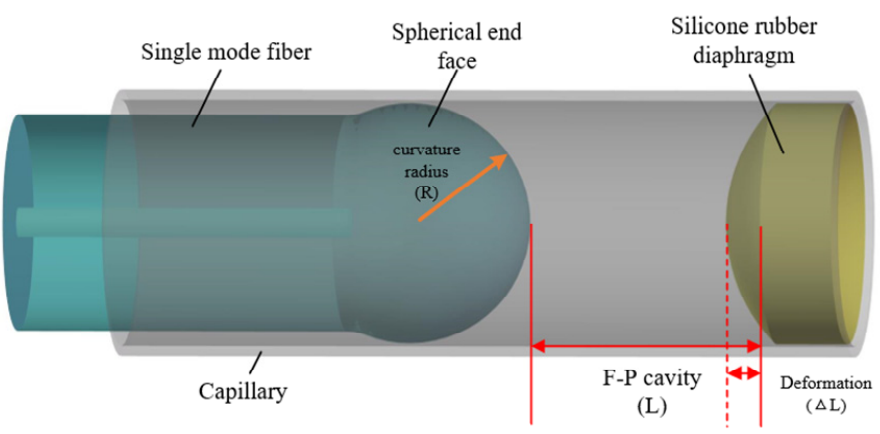

Figure 3. Structure diagram of the proposed sensor based on a spherical end face.

The sensor was composed of single-mode optical fiber with a spherical end face, a quartz capillary, and a silicone rubber diaphragm. The FP cavity was formed by the spherical end face and the inner surface of the silicone rubber. When the light is reflected 
by the silicone rubber diaphragm, more light rays will be coupled back into the fiber by the convergence effect of the spherical surface [26-28]. It also can be seen from Figure 3 that the performance of the diaphragm is related to the sensitivity of the proposed sensor and is affected by the material of silicone rubber. Therefore, further analysis is needed to optimize the parameters of the silicone rubber diaphragm to ensure high sensitivity, and to optimize the parameters of a spherical optical fiber, and initial cavity length of the sensor to improve the fringe contrast.

\subsection{Parameters Optimization of Silicone Rubber Diaphragm}

As silicone rubber diaphragms with different parameters have variable mechanical properties, static modeling is required to study the deformation of diaphragms with different parameters under different pressures. Silicone rubber consists of components $\mathrm{A}$ and $\mathrm{B}$. The main component of $\mathrm{A}$ is the silicone resin, which has good insulation, temperature resistance, and water resistance; Component $\mathrm{B}$ is mainly composed of a crosslinking agent and curing catalyst. The silicone contained in component A will affect the crosslinking density and strength of silicone rubber. Properly increasing silicone resin can significantly improve the actual mechanical properties of silicone rubber formed after curing. The crosslinking agents contained in component B can effectively improve the tear strength of silicone rubber. The mass ratio of components A and B will affect the mechanical properties of the cured material. Therefore, the mass ratio is an important factor affecting the performance of silicone rubber diaphragms [29].

To optimize the performance of silicone rubber diaphragm, the Mooney-Rivlin model, which is suitable for analyzing hyper elastomers with incompressibility and high deformation, was used to describe the mechanical properties of the silicone rubber [30].

Assume the deformation of the silicone rubber is less than $150 \%$; here, the MooneyRivlin model can be simplified with two parameters, and the relationship between stress value and the tensile ratio is derived, according to [31], as follows:

$$
\frac{\sigma}{2\left(k-1 / k^{2}\right)}=C_{10}+C_{01} / k
$$

where $\sigma$ refers to the stress value, and $k$ is the tensile ratio. Additionally, the tensile ratio $k$ is defined as

$$
k=\frac{L+\Delta L}{L}
$$

where $L$ refers to the initial length of the silicone rubber sample, and $\Delta L$ refers to the deformation.

From Equation (6), it can be derived that $C_{10}$ and $C_{01}$ coefficients affect the deformation performance of silicone rubber. To determine these two coefficients, three samples, which have the same size of $25 \mathrm{~mm} \times 6 \mathrm{~mm} \times 3.6 \mathrm{~mm}$, with a ratio of components $A$ and $B$ of $6: 1$, $8: 1$, and 10:1, respectively, were prepared and tested by the uniaxial tensile test.

Relationship between tensile ratio $(1 / k)$ and stress $\sigma /(2(k-1 k 2))$ obtained from the tensile test was plotted, which is shown in Figure 4. According to Equation (6), the slope and intercept of the fitted line function were extracted to calculate $C_{10}$ and $C_{01}$ coeeficients. The results are shown in Table 1.

Table 1. Elastic coefficients of the silicone rubber.

\begin{tabular}{ccc}
\hline Mass Ratio (M) & $\boldsymbol{C}_{\mathbf{1 0}}$ & $\boldsymbol{C}_{\mathbf{0 1}}$ \\
\hline $6: 1$ & 0.18419 & -0.12967 \\
$8: 1$ & 0.28905 & -0.14036 \\
$10: 1$ & 0.42135 & -0.15127 \\
\hline
\end{tabular}




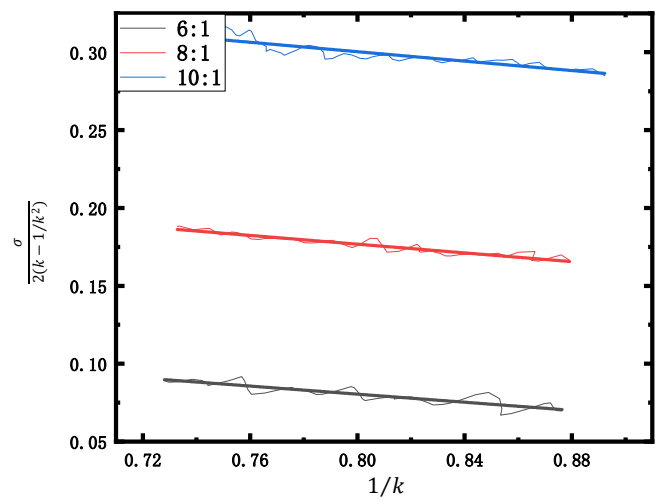

Figure 4. Relationship between tensile ratio and stress.

As the relationship between pressure and deformation of silicone rubber diaphragm cannot be expressed directly by formula, the measured parameters $C_{10}$ and $C_{01}$ were used in a model built in ANSYS for simulation, as shown in Figure 5a. To simulate the situation of silicone rubber in the capillary, we set silicone rubber diaphragms with different diameters and thicknesses, and the boundary between the silicone rubber diaphragm and the inner surface of the capillary was fixed. The pressure range was set at $0 \sim 50 \mathrm{kPa}$. Mesh generation of the model is shown in Figure $5 b$.
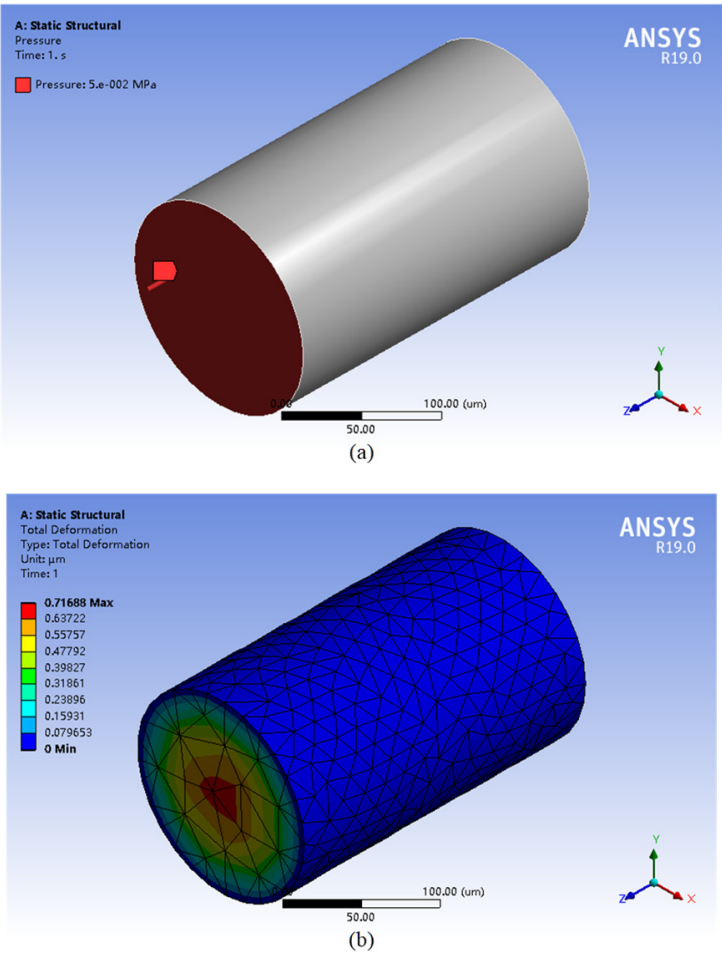

Figure 5. Model building diagram: (a) simulation model diagram and (b) deformation cloud diagram.

The relationship between the pressure and deformation are plotted in Figure 6, with different thickness $(\mathrm{H})$, diameter $(\mathrm{D})$, and mass ratio $(\mathrm{M})$. For example, when $\mathrm{M}=6: 1$ and $\mathrm{H}=200$, with increasing diaphragm diameter, the deformation range slightly increases. When $M=6: 1$, and $D=150 \mu \mathrm{m}$, with increasing diaphragm thickness, deformation range increases. When $\mathrm{H}$ and $\mathrm{D}$ are fixed, the deformation range increases greatly. These results indicate that the mass ratio is a key parameter affecting the deformation performance of the silicone rubber diaphragm. Adjusting the mass ratio is an effective way to change the sensitivity of the silicone rubber diaphragm. It also can be seen that the deformation range of silicone rubber diaphragm under the pressure of $0 \sim 50 \mathrm{kPa}$ is around several micros, 
which shows a better sensitivity, compared with reported diaphragms made by other elastic materials [18-20].
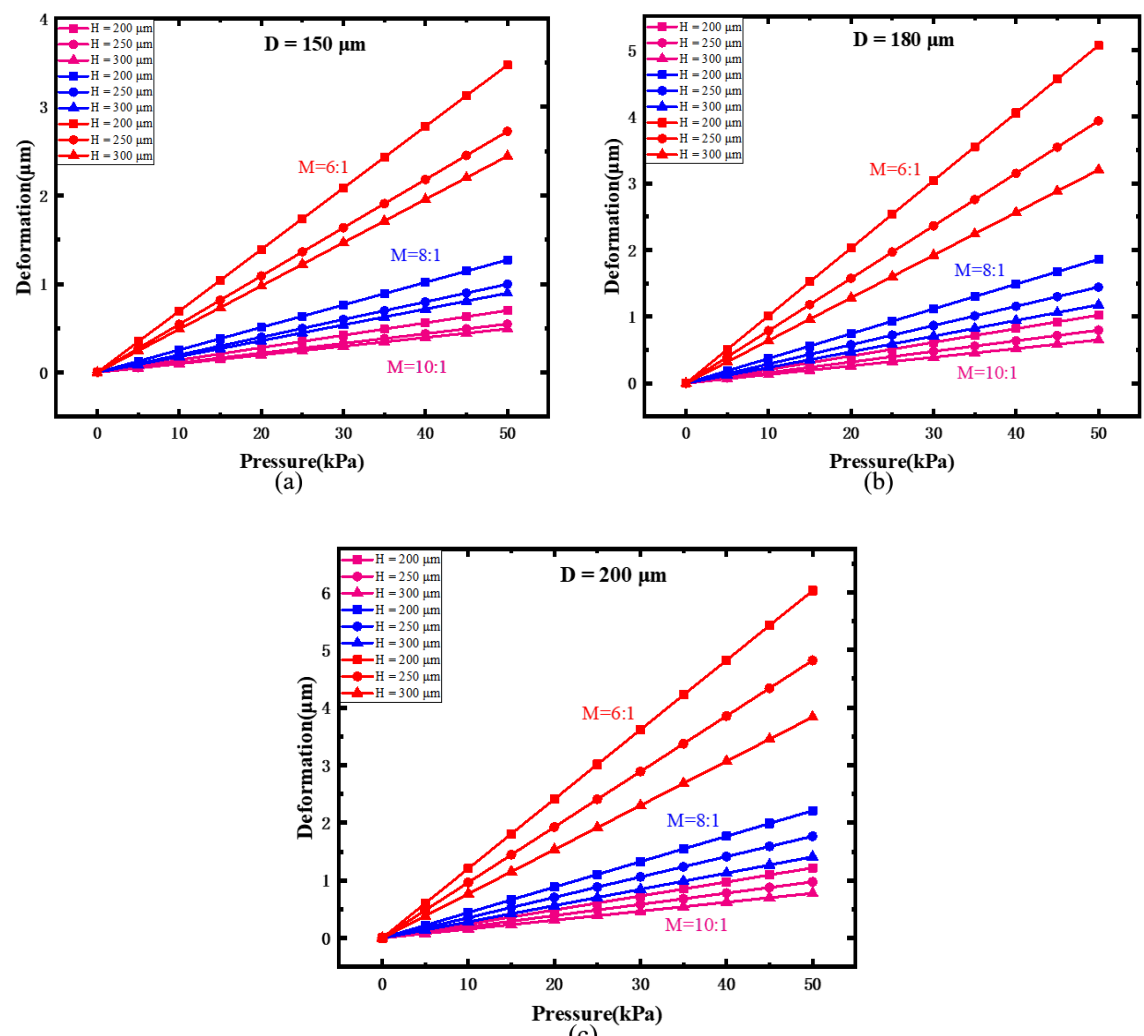

(c)

Figure 6. Relationship between the pressure and deformation: (a) diameter of $150 \mu \mathrm{m}$, (b) diameter of $180 \mu \mathrm{m}$, and (c) diameter of $200 \mu \mathrm{m}$.

In fact, an excessive amount of the curing catalyst of component B will affect the reliability of the sensor and increase the complexity of the sensor fabrication process, so the parameters of mass ratio were selected to be $8: 1$. If the thickness of the diaphragm is too thin, the connection between the diaphragm and capillary will be weak, which may cause the slippage of the diaphragm in the capillary. If it is too thick, the sensitivity of the diaphragm will be reduced. Therefore, the thickness was selected to be $250 \mu \mathrm{m}$. A diameter of $150 \mu \mathrm{m}$ of a capillary is too small for inserting a spherical end face fiber, a diameter of $200 \mu \mathrm{m}$ is slightly large for sealing the gap between the capillary and the fiber. Therefore, the diameter of the capillary was selected to be $180 \mu \mathrm{m}$.

\subsection{Optimization of the Spherical End Face}

As the spherical end face is similar to the convex lens, its curvature radius and FP cavity length will affect the intensity of the final coupling efficiency, and therefore, the structural parameters of the sensor need to be optimized to achieve a better fringe contrast of interference pattern.

The physical model of the silicone rubber pressure sensor established in ZEMAX is shown in Figure 7a, and parameters are given in Figure 7b. It can be seen that the FP cavity length change is equal to the initial cavity length minus the deformation of the silicone rubber diaphragm in the axial direction. Therefore, the pressure applied on the diaphragm was calculated by the deformation of the diaphragm in the axial direction, according to their relationship described by the results given in Figure 6. 


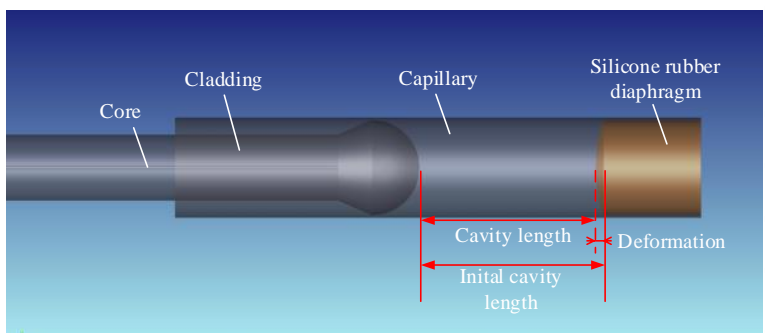

(a)

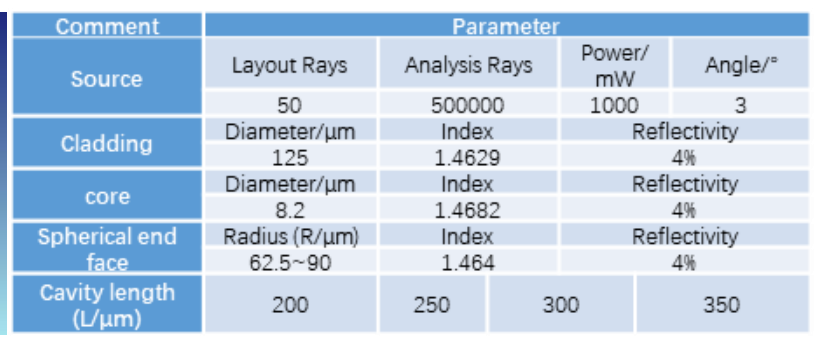

(b)

Figure 7. A 3D diagram of simulation model in ZEMAX. (a) the physical model of the silicone rubber pressure sensor; (b) simulation parameters.

Ray tracing in ZEMAX could simulate the interference results for only one single wavelength at a time, shown in Figure 8a. The total interference intensity within the range of $1525 \sim 1565 \mathrm{~nm}$ was added by every simulation result of a single wavelength, shown in Figure 8b.

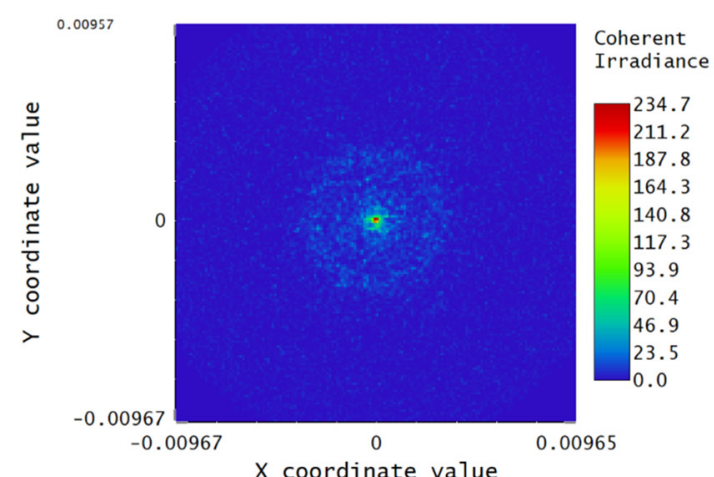

(a)

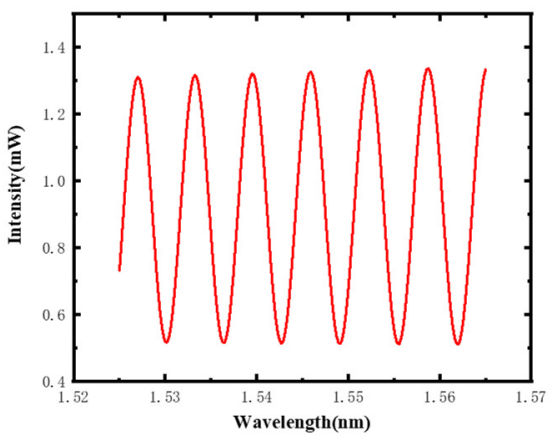

(b)

Figure 8. Ray tracing and wavelength scanning results. (a) simulation result for one single wavelength, (b) spectrum formed by simulation results of every wavelength within the range of 1525 1565 nm.

The relationship between the fringe contrast and applied pressure was simulated with different initial cavity lengths $L$ and curvature radii $R$, which is shown in Figure 9.

When the initial cavity $\mathrm{L}$ is fixed, with an increase in $\mathrm{R}$, the fringe contrast increases and then decreases. The turning point is $90 \mu \mathrm{m}$. The fringe contrast is greatly improved with the increase in the curvature radius of the spherical end face $R$. When the curvature radius $R$ is $90 \mu \mathrm{m}$, the signal contrast is the largest, which is about $4 \mathrm{~dB}$ higher than curvature radius $R$ is $62.5 \mu \mathrm{m}$. With an increase in initial cavity length $\mathrm{L}$, the fringe contrast only has a slight change. When the curvature radius $\mathrm{R}$ is $90 \mu \mathrm{m}$, the fringe contrast is slightly increased. In summary, the spherical end face can improve the fringe contrast. The best radius of the spherical end face is $90 \mu \mathrm{m}$.

According to simulation results, the structural parameters of the sensor are selected as follows: the inner diameter of quartz capillary is $180 \mu \mathrm{m}$, the radius of silicone rubber diaphragm is $90 \mu \mathrm{m}$, and the thickness is $250 \mu \mathrm{m}$. 


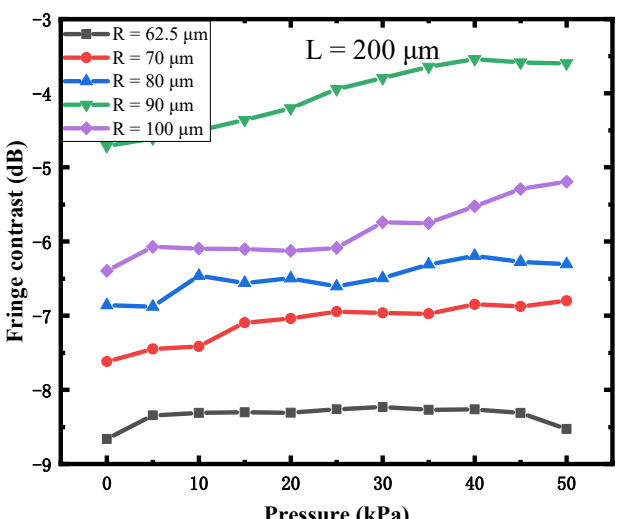

(a)

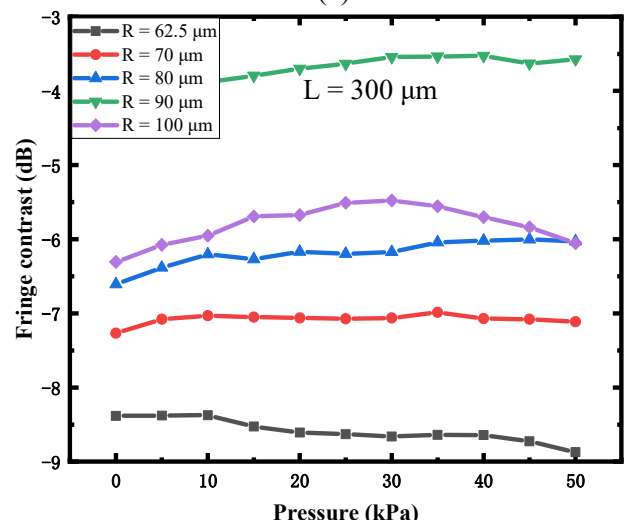

(c)

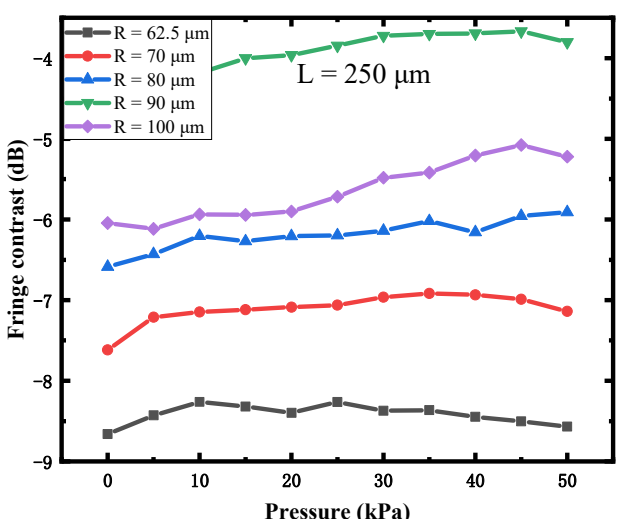

(b)

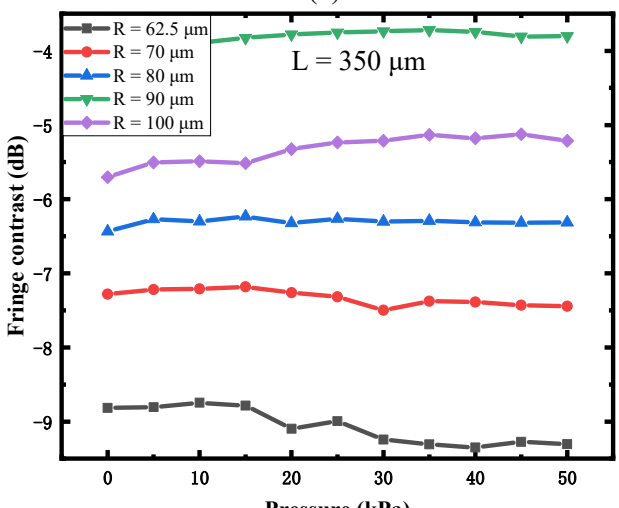

(d)

Figure 9. Relationship between the fringe contrast and applied pressure under different initial cavity lengths: (a) $\mathrm{L}=200 \mu \mathrm{m},(\mathbf{b}) \mathrm{L}=250 \mu \mathrm{m},(\mathbf{c}) \mathrm{L}=300 \mu \mathrm{m}$, and (d) $\mathrm{L}=350 \mu \mathrm{m}$.

\section{Sensor Fabrication}

To fabricate the proposed pressure sensor, we used a fusion splicer (FITEL, type: S179) to melt a fiber end face to form a spherical ball. Fusion time and discharge intensity were adjusted, and after multiple discharges, we obtained spherical balls with different radii, which are shown in Figure 10.

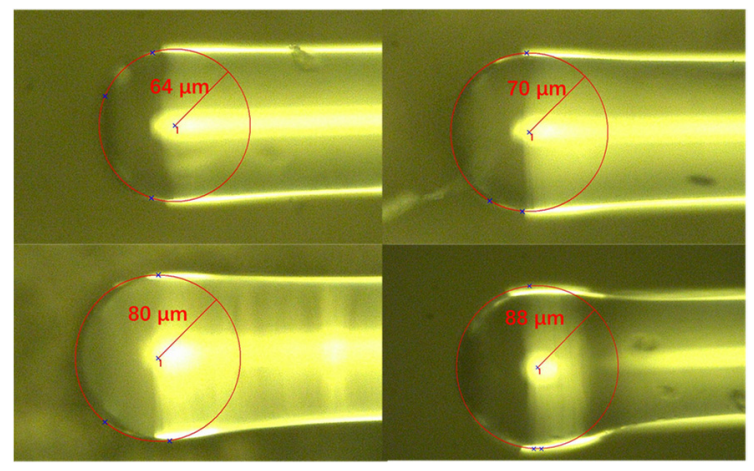

Figure 10. Spherical end faces with different radii.

Then, we began to fabricate a silicone rubber diaphragm; the fabrication process is given in Figure 11. First, silicone rubber components A and B were prepared, according to a mass ratio of 8:1; a small amount of liquid silicone rubber material was dipped onto the optical fiber, and the optical fiber was pushed forward by controlling the motor of the fusion splicer, and the liquid rubber was sucked into a quartz capillary by capillarity; then, the fiber was withdrawn back. The liquid rubber was allowed to cure in the quartz capillary for $24 \mathrm{~h}$. A fiber with a spherical end fiber was then inserted into the capillary and 
sealed with quick adhesive at the end of the capillary. The sensor sample made according to this fabrication process is shown in Figure 12.

1
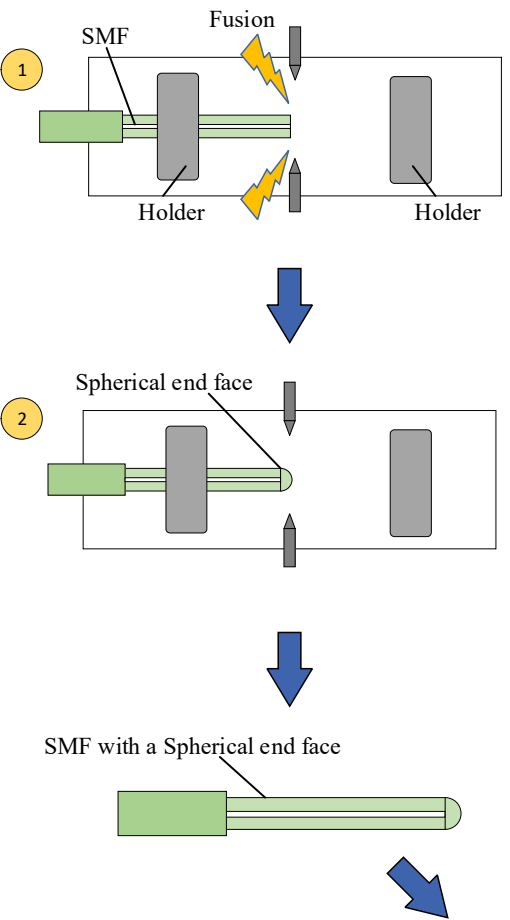

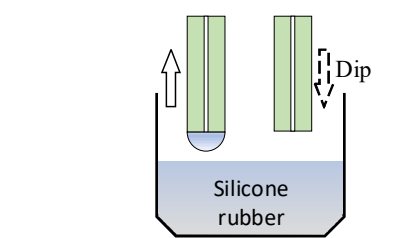

rubber

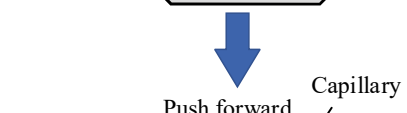

Push forwa

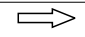

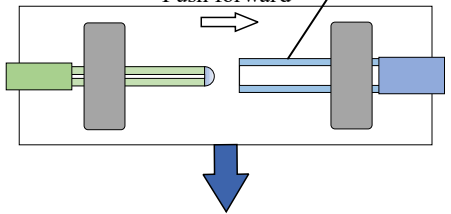

(4)

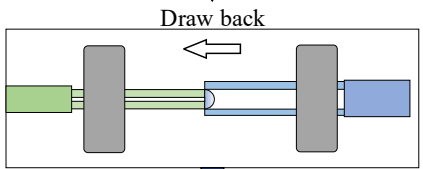

5

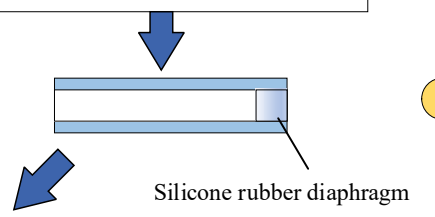

6

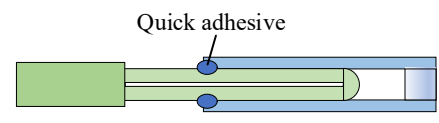

(7)

Figure 11. The fabrication process of proposed sensor.
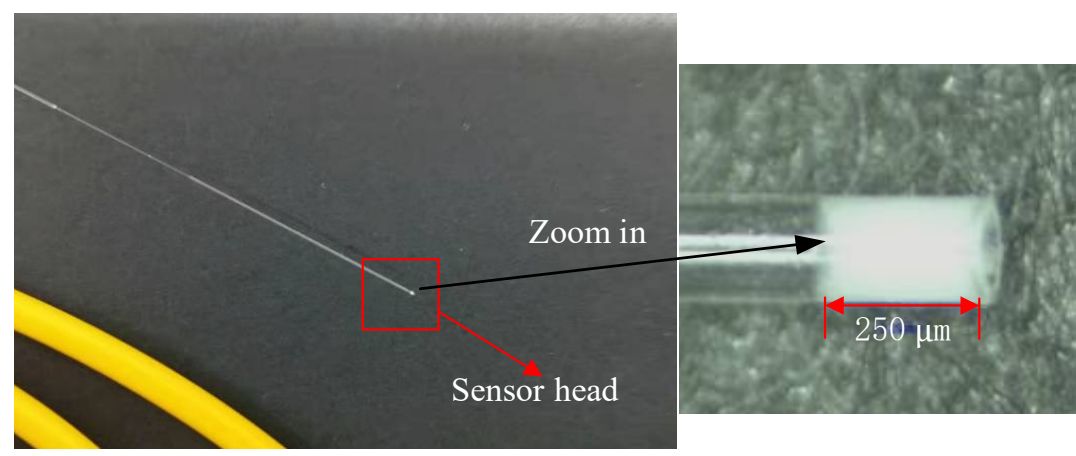

Figure 12. A pressure sensor sample.

Four pressure sensors with different curvature radii of spherical end faces were fabricated, and their interference spectra are given in Figure 13. As the curvature radius increases, the fringe contrast indeed increases. The signal-to-noise ratio is greatly improved because the noise levels progressively decrease. When the curvature radius of the spherical end face is $90 \mu \mathrm{m}$, the fringe contrast is around $20 \mathrm{~dB}$. Results are consistent with the simulation. 


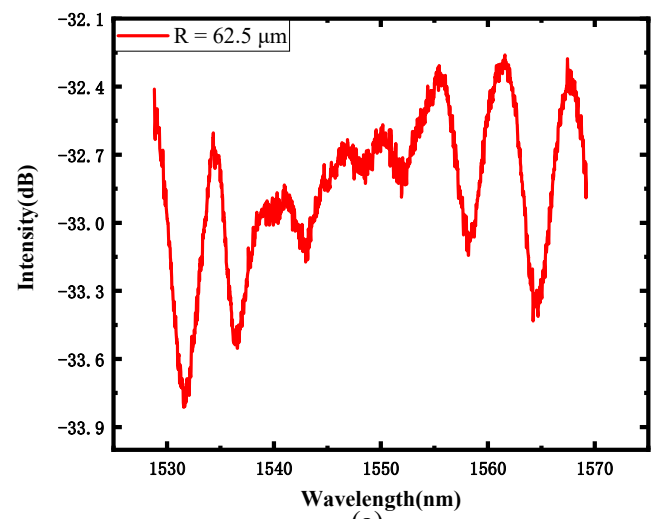

(a)

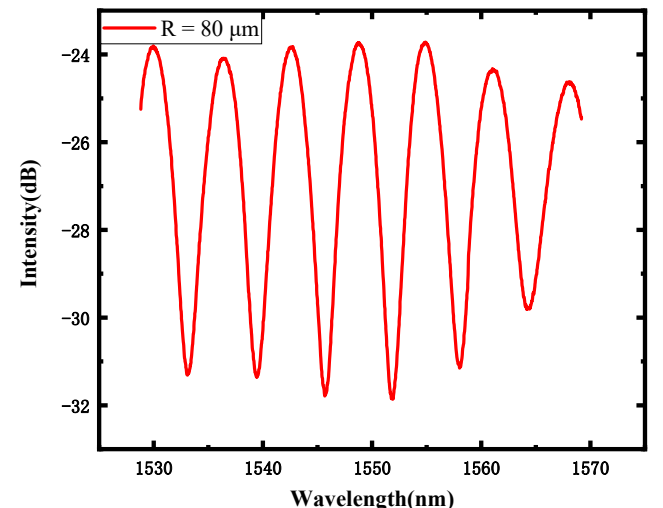

(c)

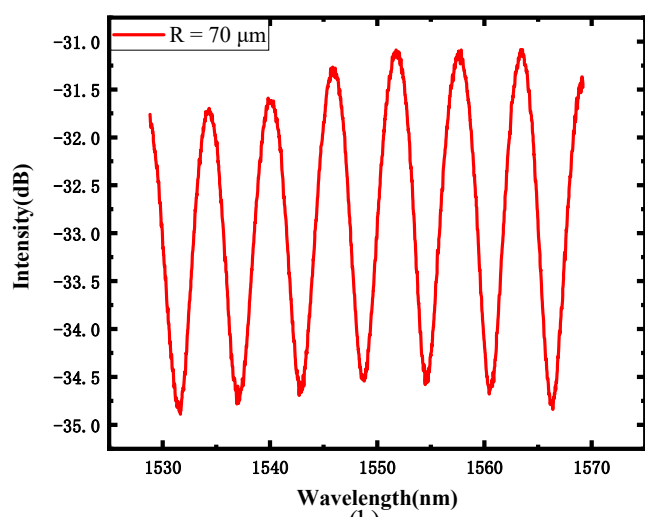

(b)

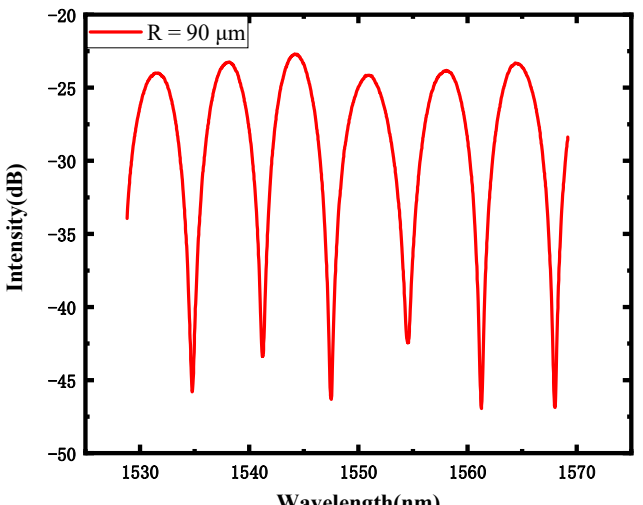

(d)

Figure 13. Sensors' spectra: (a) radius is $62.5 \mu \mathrm{m}$, (b) radius is $70 \mu \mathrm{m}$, (c) radius is $80 \mu \mathrm{m}$, and (d) radius is $90 \mu \mathrm{m}$.

\section{Experiments and Results}

To test the pressure response of our proposed sensor, an experiment system was set up, as shown in Figure 14. A proposed sensor sample was mounted to an air pressure generator (AILEIKE, ALKC400D); the pressure range is $-100 \sim 100 \mathrm{kPa}$, and the accuracy is $10 \mathrm{~Pa}$ by a homemade connector converter. The fiber pigtail of the sensor was then connected to an optical spectrum analyzer, which had a build-in broadband light source in the range of 1520 1560 nm with a wavelength resolution of $10 \mathrm{pm}$.

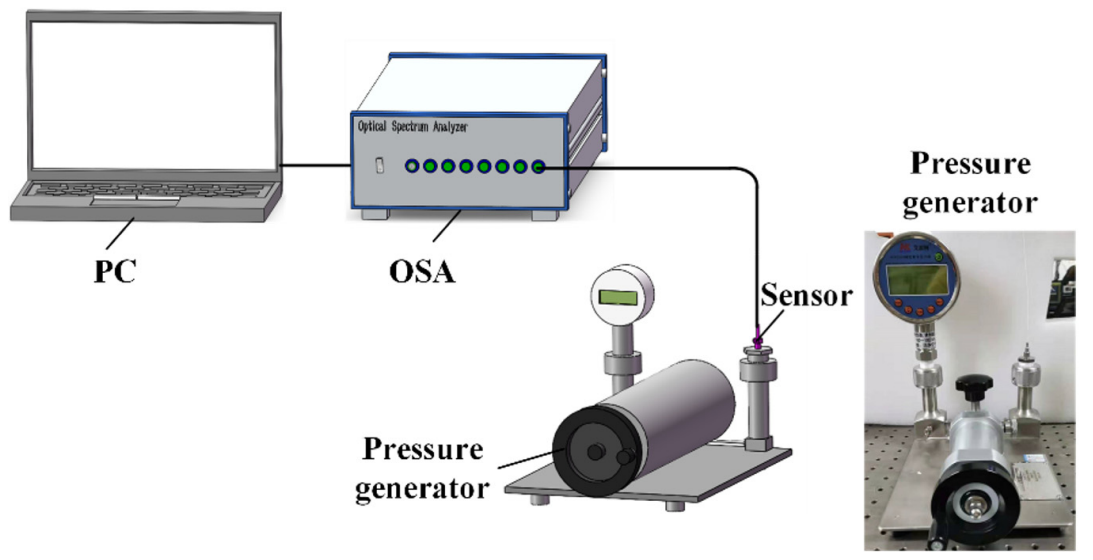

Figure 14. Experimental setup for pressure test.

The experiments were conducted three times by increasing and decreasing the pressure in the range of $0 \sim 40 \mathrm{kPa}$ with a step of $5 \mathrm{kPa}$. The pressure results are plotted in Figure 15. Figure $15 \mathrm{c}$ shows the spectrum changes during the pressure loading process. Figure $15 \mathrm{a}, \mathrm{b}$ 
show the relationship between the cavity length change and pressure in uploading and downloading processes, respectively. To analyze the pressure response properties of the sensor, a linear fitting was taken according to the results given in Figure 15a,b. We can see that our proposed senor has good linearity in the pressure response with an $\mathrm{R}^{2}$ value of 0.998 and a sensitivity of $-154.56 \mathrm{~nm} / \mathrm{kPa}$, which is around 100 times larger than reported results [20].

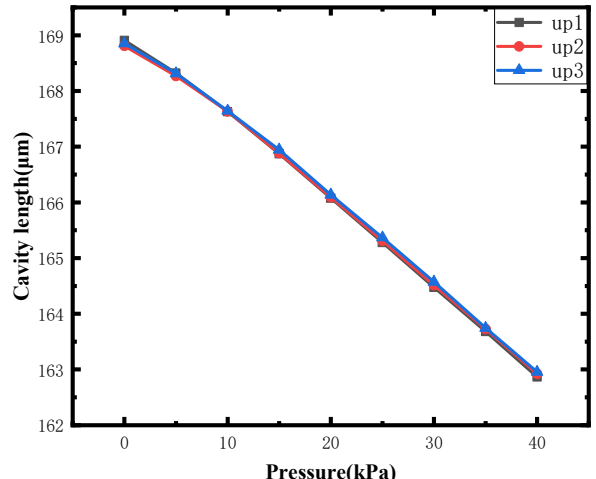

(a)

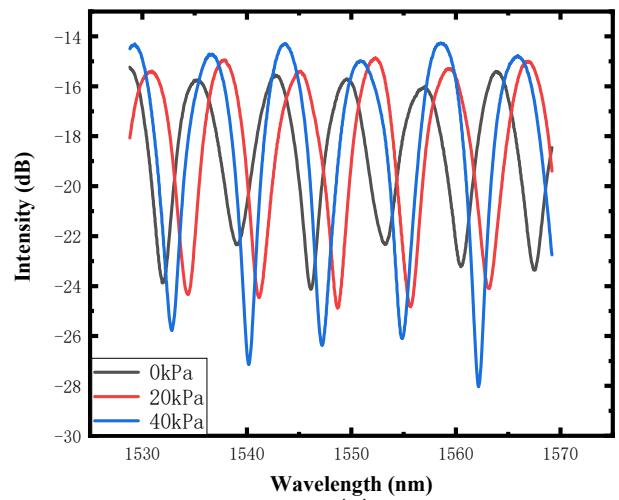

(c)

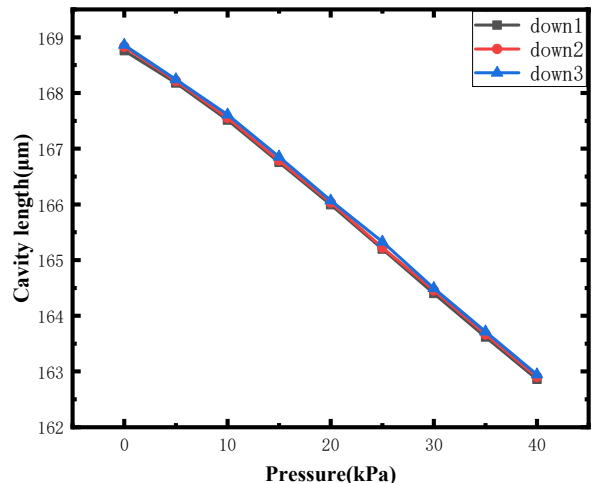

(b)

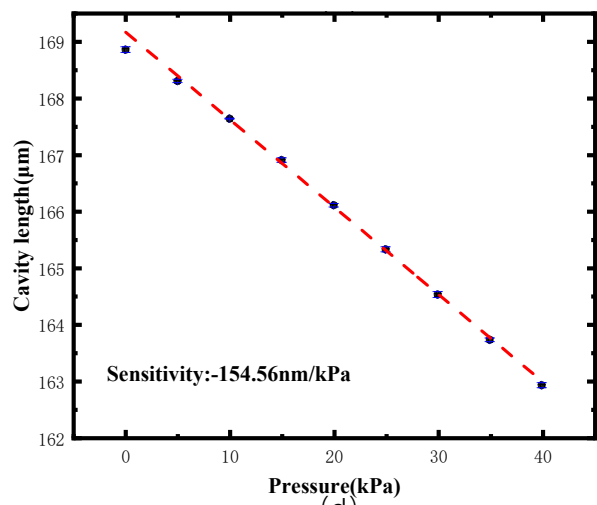

(d)

Figure 15. Response of FP cavity length to pressure. (a) relationship between the cavity length change and pressure in uploading process, (b) relationship between the cavity length change and pressure in downloading process, (c) spectrum changes during the pressure loading process, (d) a linear fitting of experimental results.

To investigate the long-term stability of the proposed sensor, an experiment was carried out in the pressure range of $0 \sim 10 \mathrm{kPa}$, during holding the pressure for $10 \mathrm{~min}$ under each spectrum data recording. The test results are shown in Figure 16. Then, we repeated the test again after 7 days, and the results are presented in Figure 17. The combined results of Figures 16 and 17 reveal that the sensor has good stability and repeatability.

The water bath method was used to investigate the response of the sensor to the change in temperature. During raising the temperature from $20^{\circ} \mathrm{C}$ to $50{ }^{\circ} \mathrm{C}$ in a water bath, sensor spectra were recorded, as shown in Figure 18. When the temperature increases, although the silicone rubber expands, the cavity length decreases, and the temperature sensitivity is $-21.36 \mathrm{~nm} /{ }^{\circ} \mathrm{C}$. Its corresponding pressure measurement error caused by temperature is around $0.143 \mathrm{kPa} /{ }^{\circ} \mathrm{C}$, which is far lower than the results reported in previous studies [20]. 


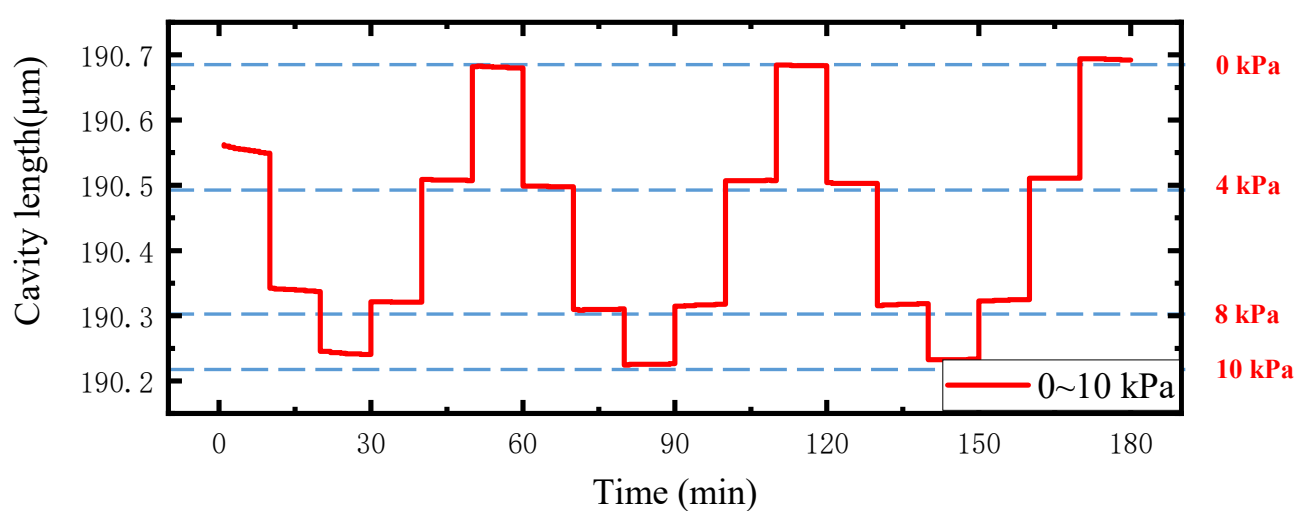

Figure 16. Stability test results.

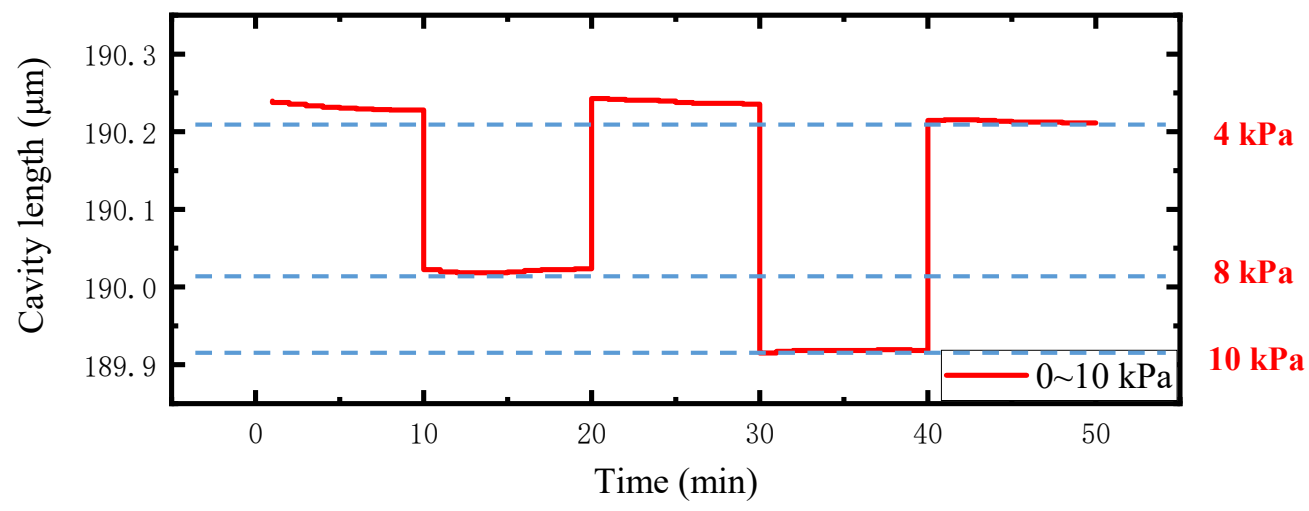

Figure 17. Test results after 7 days.

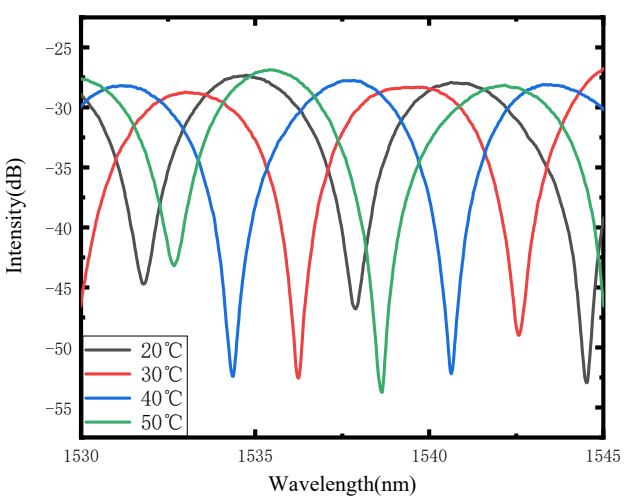

(a)

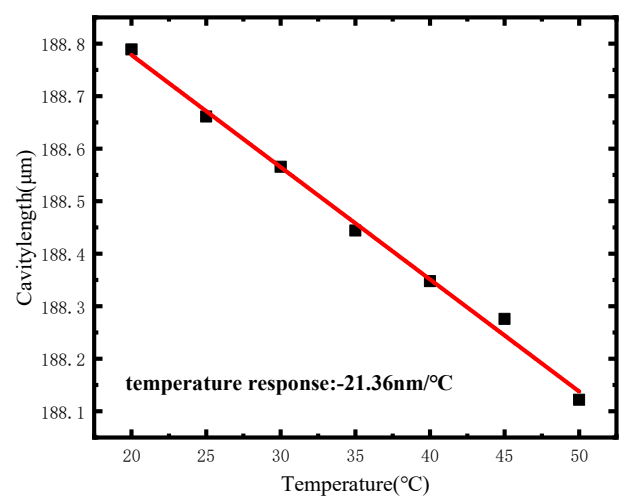

(b)

Figure 18. Temperature test results. (a) Temperature change response spectra and (b) temperature response.

\section{Conclusions}

In summary, a silicone rubber FP pressure sensor based on a spherical optical fiber end face was designed. The parameters of the proposed sensor were optimized by investigating the ratio of silicone rubber ingredients, the diameter, and thickness of the silicone rubber diaphragm, the radius of a spherical optical fiber, and initial cavity length. The spectra of fabricated samples show that our proposed sensors with a $90 \mu \mathrm{m}$ radius spherical fiber end face have a good fringe contrast.

Gas pressure experimental results within a pressure range of $0 \sim 40 \mathrm{kPa}$ show that the average sensitivity of the sensor is $-154.56 \mathrm{~nm} / \mathrm{kPa}$, and repeatability error is less than $0.71 \%$. Long-term pressure experimental results show that it has good repeatability and stability. Temperature experimental results show that its temperature cross-sensitivity is 
$0.143 \mathrm{kPa} /{ }^{\circ} \mathrm{C}$. The good performance of the proposed FP sensor will expand its applications to biochemical applications, especially human body pressure monitoring.

Author Contributions: This research was conceptualized by X.L. (Xiaohua Lei). The analysis, experiment and data processing were implemented by C.J., Y.C. and S.L. Background and potential application prospect were investigated by X.L. (Xianming Liu) and P.Z. In addition, C.J. wrote the first draft of this paper. This paper was reviewed and edited by X.L. (Xiaohua Lei), C.J., X.L. (Xianming Liu) and P.Z. All authors have read and agreed to the published version of the manuscript.

Funding: This research was supported, in part, by the National Natural Science Foundation of China (Grant No. 52175530, NO. 51975077, NO. 51675068, NO. 61875023).

Institutional Review Board Statement: Not applicable.

Informed Consent Statement: Not applicable.

Data Availability Statement: Not applicable.

Conflicts of Interest: The authors declare no conflict of interest.

\section{References}

1. Li, W.C.; Yuan, Y.G.; Yang, J.; Yuan, L.B. In-fiber integrated high sensitivity temperature sensor based on long Fabry-Perot resonator. Opt. Express 2019, 27, 14675-14683. [CrossRef] [PubMed]

2. Monteiro, C.S.; Ferreira, M.S.; Silva, S.O.; Kobelke, J.; Schuster, K.; Bierlich, J.; Frazao, O. Fiber Fabry-Perot Interferometer for Curvature Sensing. Photonic Sens. 2016, 6, 339-344. [CrossRef]

3. Zhang, Y.; Wang, K.N.; Chen, H.B.; Chen, Q.Q.; Wang, W. Non-Scanning Correlation Demodulation System for Compound Optical Fiber Fabry-Perot Sensors. Laser Optoelectron. Prog. 2019, 56, 130603. [CrossRef]

4. $\quad$ Chen, H.B.; Zhang, Y.; Li, Y.; Jing, X.; Yuan, S.Z.; Zhang, X.X.; Wang, W.; Liu, R.; Guo, Q.M. Squared peak-to-peak algorithm for the spectral interrogation of short-cavity fiber-optic Fabry-Perot sensors. Appl. Opt. 2020, 59, 1198-1205. [CrossRef]

5. Poeggel, S.; Tosi, D.; Duraibabu, D.; Leen, G.; McGrath, D.; Lewis, E. Optical Fibre Pressure Sensors in Medical Applications. Sensors 2015, 15, 17115-17148. [CrossRef]

6. Leal-Junior, A.G.; Marques, C. Diaphragm-Embedded Optical Fiber Sensors: A Review and Tutorial. IEEE Sens. J. 2021, 21, 12719-12733. [CrossRef]

7. Correia, R.; James, S.; Lee, S.W.; Morgan, S.P.; Korposh, S. Biomedical application of optical fibre sensors. J. Opt. 2018, 20, 073003-073027. [CrossRef]

8. $\quad$ Song, P.; Ma, Z.; Ma, J.; Yang, L.; Wei, J.; Zhao, Y.; Zhang, M.; Yang, F.; Wang, X. Recent Progress of Miniature MEMS Pressure Sensors. Micromachines 2020, 11, 56. [CrossRef]

9. Zhou, W.; Hou, W.; Zhang, H. Development and Application of Fiber-optic Pressure Sensor in Medical Field. Zhongguo Yi Liao Qi Xie Za Zhi Chin. J. Med. Instrum. 2018, 42, 354-356.

10. Liu, S.; Wang, Y.; Liao, C.; Wang, Y.; He, J.; Fu, C.; Yang, K.; Bai, Z.; Zhang, F. Nano silica diaphragm in-fiber cavity for gas pressure measurement. Sci. Rep. 2017, 7, 787-795. [CrossRef]

11. Guo, X.; Zhou, J.; Du, C.; Wang, X. Highly Sensitive Miniature All-Silica Fiber Tip Fabry-Perot Pressure Sensor. IEEE Photonics Technol. Lett. 2019, 31, 689-692. [CrossRef]

12. Ma, J.; Jin, W.; Ho, H.L.; Dai, J.Y. High-sensitivity fiber-tip pressure sensor with graphene diaphragm. Opt. Lett. 2012, 37, 2493-2495. [CrossRef] [PubMed]

13. Huang, B.; Wang, Y.; Mao, C. Temperature-Independent Gas Pressure Sensor with High Birefringence Photonic Crystal Fiber-Based Reflective Lyot Filter. Sensors 2019, 19, 5312. [CrossRef] [PubMed]

14. Kim, J.T.; In-Gyoo, K. Flexible and Wearable Pressure Sensor for Biomedical Applications. J. Korean Inst. Commun. Inf. Sci. 2018, $43,1694-1702$.

15. Sikarwar, S.; Singh, S.S.; Yadav, B.C. Review on Pressure Sensors for Structural Health Monitoring. Photonic Sens. 2017, 7, 294-304. [CrossRef]

16. Leal, A.L.; Damasceno, C.A.; Soares, G.F.; Pinheiro, G.R.; Bezerra, L.D.; Prado, M.D.P.; Perci, Y.M.; Neto, M.L.D. Low cost volume sensors for mechanical ventilators. IEEE Lat. Am. Trans. 2021, 19, 1083-1089.

17. Farooq, M.; Iqbal, T.; Vazquez, P.; Farid, N.; Thampi, S.; Wijns, W.; Shahzad, A. Thin-Film Flexible Wireless Pressure Sensor for Continuous Pressure Monitoring in Medical Applications. Sensors 2020, 20, 6653. [CrossRef]

18. Wu, J.; Yao, M.; Xiong, F.; Zhang, A.P.; Tam, H.-Y.; Wai, P.K.A. Optical Fiber-Tip Fabry-Pérot Interferometric Pressure Sensor Based on an In Situ $\mu$-Printed Air Cavity. J. Lightwave Technol. 2018, 36, 3618-3623. [CrossRef]

19. Luo, C.; Liu, X.; Liu, J.; Shen, J.; Li, H.; Zhang, S.; Hu, J.; Zhang, Q.; Wang, G.; Huang, M. An Optimized PDMS Thin Film Immersed Fabry-Perot Fiber Optic Pressure Sensor for Sensitivity Enhancement. Coatings 2019, 9, 290. [CrossRef]

20. Cheng, X.; Dash, J.N.; Gunawardena, D.S.; Htein, L.; Tam, H.Y. Silicone Rubber Based Highly Sensitive Fiber-Optic Fabry-Perot Interferometric Gas Pressure Sensor. Sensors 2020, 20, 4927. [CrossRef] 
21. Wang, Q.H.; Liu, X.; Wang, D.N. Ultra-sensitive gas pressure sensor based on vernier effect with controllable amplification factor. Opt. Fiber Technol. 2021, 61, 102404-102408. [CrossRef]

22. Wu, N.; Tian, Y.; Zou, X.; Zhai, Y.; Barringhaus, K.; Wang, X. A miniature fiber optic blood pressure sensor and its application in in vivo blood pressure measurements of a swine model. Sens. Actuators B Chem. 2013, 181, 172-178. [CrossRef]

23. Zhao, Y.; Chen, M.-q.; Xia, F.; Lv, R.-Q. Small in-fiber Fabry-Perot low-frequency acoustic pressure sensor with PDMS diaphragm embedded in hollow-core fiber. Sens. Actuators A Phys. 2018, 270, 162-169. [CrossRef]

24. Liang, H.; Jia, P.G.; Liu, J.; Fang, G.C.; Li, Z.; Hong, Y.P.; Liang, T.; Xiong, J.J. Diaphragm-Free Fiber-Optic Fabry-Perot Interferometric Gas Pressure Sensor for High Temperature Application. Sensors 2018, 18, 1011. [CrossRef]

25. Chen, P.; Dai, Y.T.; Zhang, D.S.; Wen, X.Y.; Yang, M.H. Cascaded-Cavity Fabry-Perot Interferometric Gas Pressure Sensor based on Vernier Effect. Sensors 2018, 18, 3677. [CrossRef] [PubMed]

26. Lei, X.H.; Xie, L.; Qi, L.S.; Chen, W.M. Coupling efficiency of light intensity from the blackbody cavity into a cone-shaped optical fiber. Appl. Opt. 2019, 58, 1707-1714. [CrossRef]

27. Lee, Y.G.; Park, C.H.; Back, S.W.; Kim, H.J.; Lee, S.S. Alignment tolerant expanded beam connector based on a gapless fiber-lens interface. Appl. Opt. 2016, 55, 341-344. [CrossRef]

28. Song, C.X.; Lei, X.H.; Xie, L.; Liu, X.M.; Chen, W.M. Coupling Efficiency of Light Intensity from Blackbody Cavity into Spherical Optical Fiber. Acta Photonica Sin. 2020, 49, 0906001. [CrossRef]

29. Hao, W.L.; Zeng, H.H.; Chen, X.Q.; He, W.; Zhou, R.; Li, J.H.; Zhang, H.X. Insights into the reinforcement and mechanism of silicone mold rubber co-modified with WCB and MMQ resin. Mater. Res. Express 2021, 8, 035201. [CrossRef]

30. Marckmann, G.; Verron, E. Comparison of Hyperelastic Models for Rubber-Like Materials. Rubber Chem. Technol. 2006, 79, 835-858. [CrossRef]

31. Liu, M.; Wang, Q.C.; Wang, G.Q. Determination of Material Constants in Rubber Mooney Rivlin Model. China Rubber Ind. 2011, $58,241-245$. 